\title{
Regional Embedding Enables High-Level Quantum Chemistry for Surface Science
}

\author{
Bryan T. G. Lau, ${ }^{1}$ Gerald Knizia, ${ }^{2, \text { a) }}$ and Timothy C. Berkelbach ${ }^{1,3, b)}$ \\ ${ }^{1)}$ Center for Computational Quantum Physics, Flatiron Institute, New York, New York 10010 USA \\ ${ }^{2)}$ Department of Chemistry, Pennsylvania State University, University Park, Pennsylvania 16802 USA \\ ${ }^{3)}$ Department of Chemistry, Columbia University, New York, New York 10027 USA
}

\begin{abstract}
Compared to common density functionals, ab initio wave function methods can provide greater reliability and accuracy, which could prove useful when modeling adsorbates or defects of otherwise periodic systems. However, the breaking of translational symmetry necessitates large supercells that are often prohibitive for correlated wave function methods. As an alternative, we introduce the regional embedding approach, which enables correlated wave function treatments of only a target fragment of interest through small, fragment-localized orbital spaces constructed using a simple overlap criterion. Applications to the adsorption of water on lithium hydride, hexagonal boron nitride, and graphene substrates show that regional embedding combined with focal point corrections can provide converged CCSD(T) (coupled cluster) adsorption energies with very small fragment sizes.
\end{abstract}

In electronic structure theory, ab initio wave function methods provide a hierarchy of approximations that allow for systematic improvability-a feature missing in density functional theory (DFT). While ab initio methods were originally limited to molecules, advances in the past decade have enabled approaches like many-body perturbation theory or coupledcluster theory to be applied to atomistic periodic systems. ${ }^{1-8}$ However, these methods are expensive and exploitation of translational symmetry is usually necessary to reduce their computational cost to manageable levels. Unfortunately, this is impossible in several applications scenarios of tremendous practical importance. For example, in models of crystal defects (e.g. for doping in semiconductor devices), or surface adsorbates (e.g. as involved in most core processes of heterogeneous catalysis), translational symmetry is broken. In practice, the non-periodic system is then described with supercells, which must be large in order to eliminate artifacts caused by the spurious interactions of the defect or adsorbate with its periodic images in other supercells. This is particularly problematic for ab initio methods, which are already expensive with translational symmetry.

However, when translational symmetry is broken by spatially-localized perturbations, the high cost of conventional treatments by large supercells is often artifical and can be avoided. To substantiate this claim, we here demonstrate that ab initio molecular adsorption energies can be obtained at significantly reduced cost, compared to the conventional supercell treatment, by a regional embedding technique. In general, the accurate prediction of noncovalent adsorption energies is challenging because they arise as small energy differences between large total energies. ${ }^{9}$ However, as opposed to total energies, adsorption energies are natural targets for regional embedding approaches, because they focus the computational effort on the important region of change. Like previous periodic embedding approaches, ${ }^{10-13}$ we only correlate orbitals that are localized to a region near the adsorbate and can converge to the supercell result by increasing the size of the region. This

\footnotetext{
a)Electronic mail: knizia@psu.edu

b)Electronic mail: tim.berkelbach@gmail.com
}

technique allows us to perform expensive post-Hartree-Fock (HF) calculations on only the chemically important piece of the system, while retaining large supercells at the mean-field level to eliminate finite-size and finite-coverage effects.

Unlike most previous embedding approaches, we localize the orbitals based upon their overlap with the fragment of interest in Hilbert space, rather than invoking real-space densities or localization functionals. The approach builds on quantum information concepts and, in particular, density matrix embedding theory (DMET). ${ }^{14-16}$ DMET gives a precise prescription for the factorization of a system's wavefunction into active and inactive components, which we leverage to define a regional embedding procedure. For the orbitals occupied in the HF determinant, we define an operator that projects onto a minimal atomic orbital (AO) basis of the atoms in the fragment $A$,

$$
\hat{P}^{\mathrm{occ}}=\sum_{\rho, \tau \in A}|\rho\rangle\left[\mathbf{S}^{-1}\right]_{\rho \tau}\langle\tau|
$$

where $\rho, \tau$ are the minimal AO basis functions on the fragment and $\mathbf{S}$ is the overlap matrix in the minimal AO basis; let $|a|$ indicate the total number of minimal AO basis functions on the fragment. The eigenvectors of $\hat{P}^{o c c}$ in the basis of canonical occupied orbitals, $P_{i j}^{\text {occ }}=\left\langle\varphi_{i}\left|\hat{P}^{\text {occ }}\right| \varphi_{j}\right\rangle$, define a rotated set of occupied orbitals whose eigenvalues quantify their overlap with the fragment space. As a unitary transformation of the occupied orbitals, this procedure does not change the HF wavefunction, but merely separates the occupied orbitals into a set with nonzero overlap on the fragment (at most $|a|$ ) and a set with zero overlap on the fragment. If we were to use the same projection operator to rotate the canonical virtual orbitals, this procedure would generate the atomic valence active space $^{17}$ of the fragment atoms. As explained by DMET, the combined active space would have at most $2|a|$ orbitals, which is the maximum number required to represent the mean-field hybridization between the fragment minimal AOs and the rest of the system. However, this small active space lacks the basis functions necessary for a description of dynamical correlation, which is critical for accurate adsorption energies. Therefore, we modify the procedure for the virtual space. We now let the operator $\hat{P}^{\text {vir }}$ project onto the computational AO basis func- 
tions of the atoms in the fragment, and denote the number of these basis functions as $|A|$. Diagonalization in the basis of the canonical virtual orbitals, $P_{a b}^{\mathrm{vir}}=\left\langle\varphi_{a}\left|\hat{P}^{\mathrm{vir}}\right| \varphi_{b}\right\rangle$, then defines a unitary transformations separating the virtuals into those with nonzero overlap on the fragment (at most $|A|$ ) and those with zero overlap on the fragment. As discussed in Refs. 17 and 18, the combined occupied and virtual orbital spaces with fragment overlap are closely related to the combined impurity and bath orbital spaces in conventional DMET.

We propose to correlate only those orbitals that have significant overlap with the fragment, defined by a threshold on the eigenvalues of the projection operators. The rotated orbitals with insufficient overlap on the fragment space are simply frozen in the post-HF calculations, making the insertion of regional embedding into existing quantum chemistry workflows straightforward. Compared to other localization procedures, the one proposed here does not require minimization of a cost function (as in Foster-Boys, ${ }^{19}$ Edmiston-Ruedenberg, ${ }^{20}$ Pipek-Mezey, ${ }^{21}$ or higher-order moment-based ${ }^{22,23}$ localization) and does not result in nonorthogonal orbitals (as in projected atomic orbitals ${ }^{23-27}$ ). We note that two recent works have explored similar overlap-based approaches to embedding, focusing on the occupied orbitals only ${ }^{28}$ and on multireference problems. ${ }^{29}$ In future work we will compare the spatial extent and convergence properties of the local orbitals of these various approaches more thoroughly.

While any high-level quantum chemistry method may be used in the fragment calculation, here we focus on secondorder Møller-Plesset perturbation theory (MP2) and coupledcluster theory with single and double excitations (CCSD) and perturbative triples $(\mathrm{CCSD}(\mathrm{T}))$. These methods are valuable in this problem area for their nonempirical treatment of dispersion interactions. Furthermore, $\operatorname{CCSD}(\mathrm{T})$ is a powerful method in molecular thermochemistry: in small and electronically benign molecules, energy differences computed at its basis set limit typically deviate by $\leq 2 \mathrm{~kJ} / \mathrm{mol}$ from the exact result, far outperforming all known DFT methods. ${ }^{30}$ The extent to which this accuracy persists for the thermochemistry of solids and surfaces remains to be seen.

To test regional embedding, we study the adsorption of a water molecule on the (001) surface of lithium hydride $(\mathrm{LiH}),{ }^{31,32}$ hexagonal boron nitride $(\mathrm{hBN}),{ }^{6,33}$ and graphene. ${ }^{34-37}$ These systems have been used to demonstrate the application of ab initio methods to periodic systems, and reference results obtained by extrapolation to the thermodynamic and complete basis set limits are available for a variety of DFT, wavefunction, and quantum Monte Carlo methods. We use literature values for the orientation and location of the water molecule; for water on graphene, we consider the socalled " 0 -leg" configuration. ${ }^{36}$ The calculations are performed with slab geometries using a single layer of graphene and hBN and two layers of the $\mathrm{LiH}(001)$ plane along with $20 \AA$ of vacuum in the direction perpendicular to the surface.

Figure 1 illustrates the method of selecting atoms in the fragment, using the example of water on graphene. The atoms of the water molecule are always included in the fragment and we add atoms from the substrate based upon their radial distance from the water molecule. The adsorption energy is

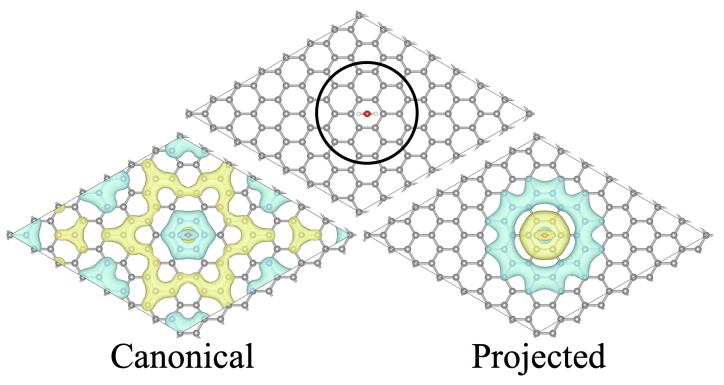

FIG. 1. An illustration of the embedding scheme used in this paper, for water on a $7 \times 7$ graphene supercell, with an example of randomly selected canonical and projected occupied orbitals. Carbon atoms from graphene are selected to be in the fragment based upon the radial distance from the oxygen atom in the water molecule.

found from three separate calculations,

$$
E_{\text {ads }}=E_{\mathrm{H}_{2} \mathrm{O}+\text { substrate }}-E_{\mathrm{H}_{2} \mathrm{O}}-E_{\text {substrate }}
$$

where the embedding procedure is applied to each calculation. We apply the counterpoise correction for basis set superposition error by performing each calculation in the full basis set of the water and substrate, ${ }^{38}$ using the same eigenvalue cutoff in each.

All calculations are performed with periodic boundary conditions as implemented in PySCF, ${ }^{39,40}$ using Gaussian density fitting ${ }^{41}$ of periodic integrals, GTH pseudopotentials, ${ }^{42}$ and their corresponding family of basis sets. We use the augTZV2P basis set for the water molecule and a mixture of the DZVP and TZVP basis sets for the substrate. We use the SVZ basis as the minimal $\mathrm{AO}$ basis required for projection of the occupied orbitals and the eigenvalue cutoff is set to 0.1 for both the occupied and virtual space, although a number of other truncation procedures exist. We are in the process of being exhaustively and systematically investigating optimum truncation procedures, which may depend on the system and properties of interest; we will publish these results elsewhere. Following truncation, we perform post-HF calculations using semicanonical orbitals obtained by diagonalizing the Fock matrix in the basis of the localized fragment orbitals.

Figure 2 plots the MP2 adsorption energy as a function of the number of substrate atoms in the fragment for water on (a) $\mathrm{LiH}$, (b) hBN, and (c) graphene for a series of supercell sizes, where we use the the DZVP basis for the substrate atoms. The reasonable cost of MP2 calculations allows us to increase the fragment size to the full supercell limit and thus carefully study the convergence. Interestingly, the convergence behaviors are quite different, reflecting the various electronic characters of the molecule-substrate interaction.

The adsorption energy of water on $\mathrm{LiH}$, Figure 2a, rapidly converges to the full supercell limit, and the energy minimally changes as the supercell size is increased from $4 \times 4$ to $6 \times 6$. These results reflect the highly local nature of the interaction of water with the highly insulating $\mathrm{LiH}$ (001) surface, which is captured at fragment sizes much smaller than the full supercell.

In contrast, the MP2 adsorption energy curves for hBN, 

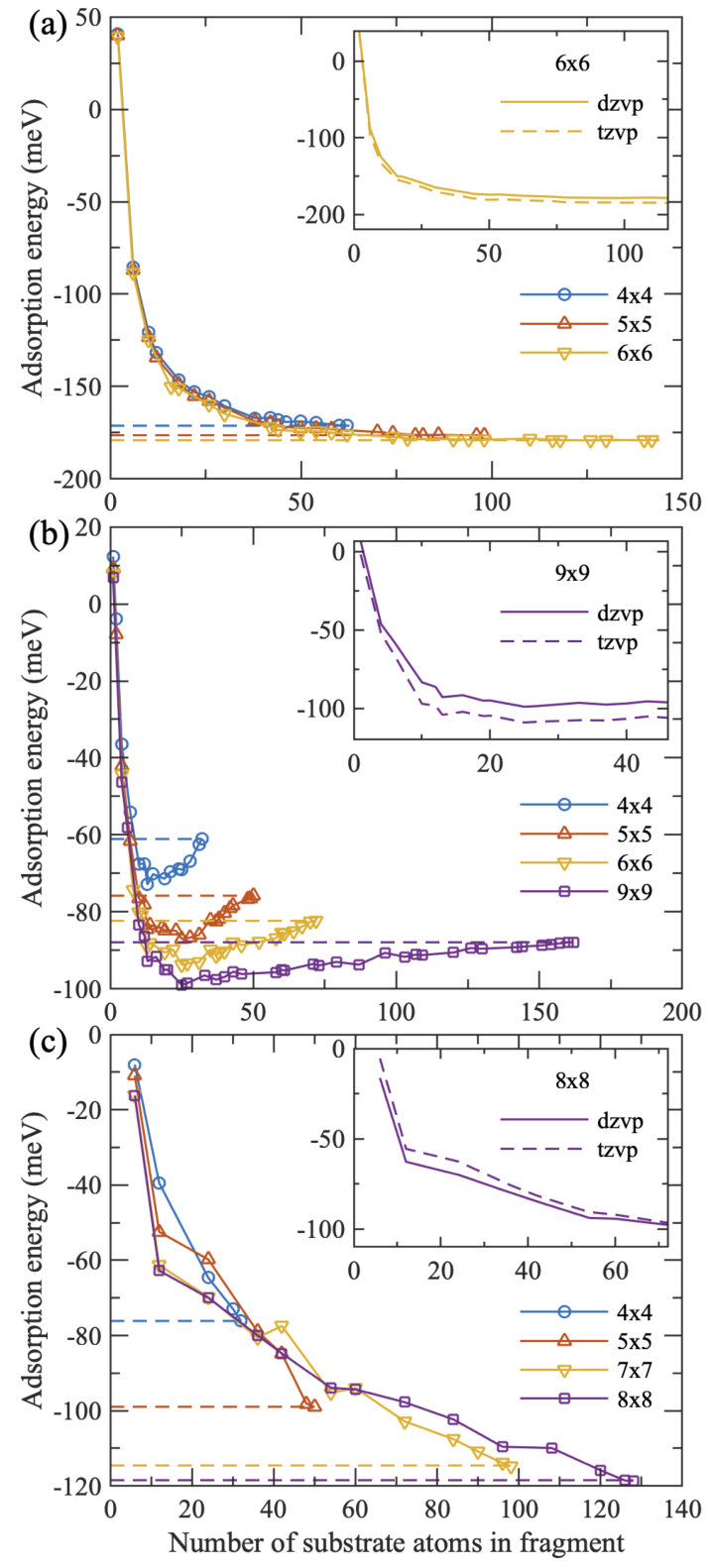

FIG. 2. The MP2 adsorption energy vs. fragment size, for water on (a) $\mathrm{LiH}$, (b) hBN, and (c) graphene, with the DZVP basis on the substrate. The insets compare the DZVP and TZVP basis sets. The smaller DZVP basis allows for MP2 calculations of all fragment sizes up to the full supercell, the results of which are indicated by a dashed horizontal line.

Figure $2 b$, quickly reach a minimum before slowly rising to the full supercell energy. It is possible that the minimum value represents the most accurate energy for a given supercell size and that the rise in energy is due to error from the finite coverage of water. In other words, as the fragment grows in size, it includes orbitals that are erroneously perturbed by the periodic images, leading to a spurious interaction that raises the adsorption energy. However, as the supercell grows in size, the water molecule is further separated from its periodic images, and the fragment size can grow larger without includ- ing these unphysical orbitals. This behavior can be seen by comparing the adsorption energy for a fixed number of atoms in the fragment, which smoothly decreases as the supercell size is increased; importantly, the MP2 step of these calculations have identical cost because the fragment size is the same, highlighting a strength of our regional embedding method.

Finally, the MP2 adsorption energy of water on graphene, Figure 2c, exhibits a very slow convergence to the the supercell limit and shows no indication of absolute convergence. This behavior is almost surely due to the challenge of localizing orbitals in gapless materials ${ }^{23,43-45}$ and the closely related importance of long-range dispersion.

The inset of each panel in Figure 2 shows a comparison to results obtained by using the TZVP basis for the substrate atoms in the fragment (and DZVP for the substrate atoms outside of the fragment). These latter calculations are limited in size by memory or convergence issues associated with linear dependencies in periodic systems. Using the larger TZVP basis on the fragment does not qualitatively alter the convergence behavior and changes the adsorption energy by $10 \mathrm{meV}$ or less.

In Figure 3, we present our main results of the adsorption energy obtained using MP2, CCSD, and $\operatorname{CCSD}(\mathrm{T})$, for the same three systems with the largest supercell sizes considered, i.e. $6 \times 6$ for $\mathrm{LiH}, 9 \times 9$ for $\mathrm{hBN}$, and $8 \times 8$ for graphene. As in the insets of Figure 2, all calculations use the TZVP basis for the substrate atoms belonging to the fragment and DZVP for the remainder, except for the $(\mathrm{T})$ correction, which used the DZVP basis for all substrate atoms,

$$
\begin{aligned}
E_{\mathrm{CCSD}(\mathrm{T})}(N, \mathrm{TZ}) & =E_{\mathrm{CCSD}}(N, \mathrm{TZ})+\delta^{(\mathrm{T})}(N, \mathrm{DZ}), \\
\delta^{(\mathrm{T})} & =\left[E_{\mathrm{CCSD}(\mathrm{T})}-E_{\mathrm{CCSD}}\right](N, \mathrm{DZ}),
\end{aligned}
$$

where $N$ is the number of fragment atoms and $E$ is the adsorption energy. The adsorption energies, plotted with solid lines and filled symbols, follow the same shape and rate of convergence as the MP2 results presented in Figure 2. In particular, the shape of convergence correlates with the adsorption energy, as seen when comparing the strong, local adsorption of water on $\mathrm{LiH}$ versus the dispersion-dominated interaction with $\mathrm{hBN}$ and graphene.

Given the similar convergence behavior of the adsorption energies and the relatively cheap cost of MP2, we can apply a simple finite-size (FS) correction,

$$
\begin{aligned}
E(\infty, \mathrm{TZ}) & =E(N, \mathrm{TZ})+\delta^{\mathrm{FS}}(N, \mathrm{DZ}) \\
\delta^{\mathrm{FS}}(N, \mathrm{DZ}) & =\left[E_{\mathrm{MP} 2}(\infty)-E_{\mathrm{MP} 2}(N)\right](\mathrm{DZ}),
\end{aligned}
$$

where we estimate $E_{\mathrm{MP} 2}(\infty)$, the MP2 adsorption energy in the thermodynamic limit by a $1 / N$ extrapolation of a series of full supercell MP2 calculations (see the dashed lines in Figure 2). Although previous work has used a $1 / N^{2}$ extrapolation of the adsorption energy, we find our data fits much better to $1 / N$ (shown in the Supporting Information). The finite-size corrected data are frequently converged to within $10 \mathrm{meV}$ when correlating only five to ten atoms in the substrate! We note that the finite-size correction can also be viewed as a focalpoint basis set correction, accounting for the frozen orbitals in the supercell calculation. 

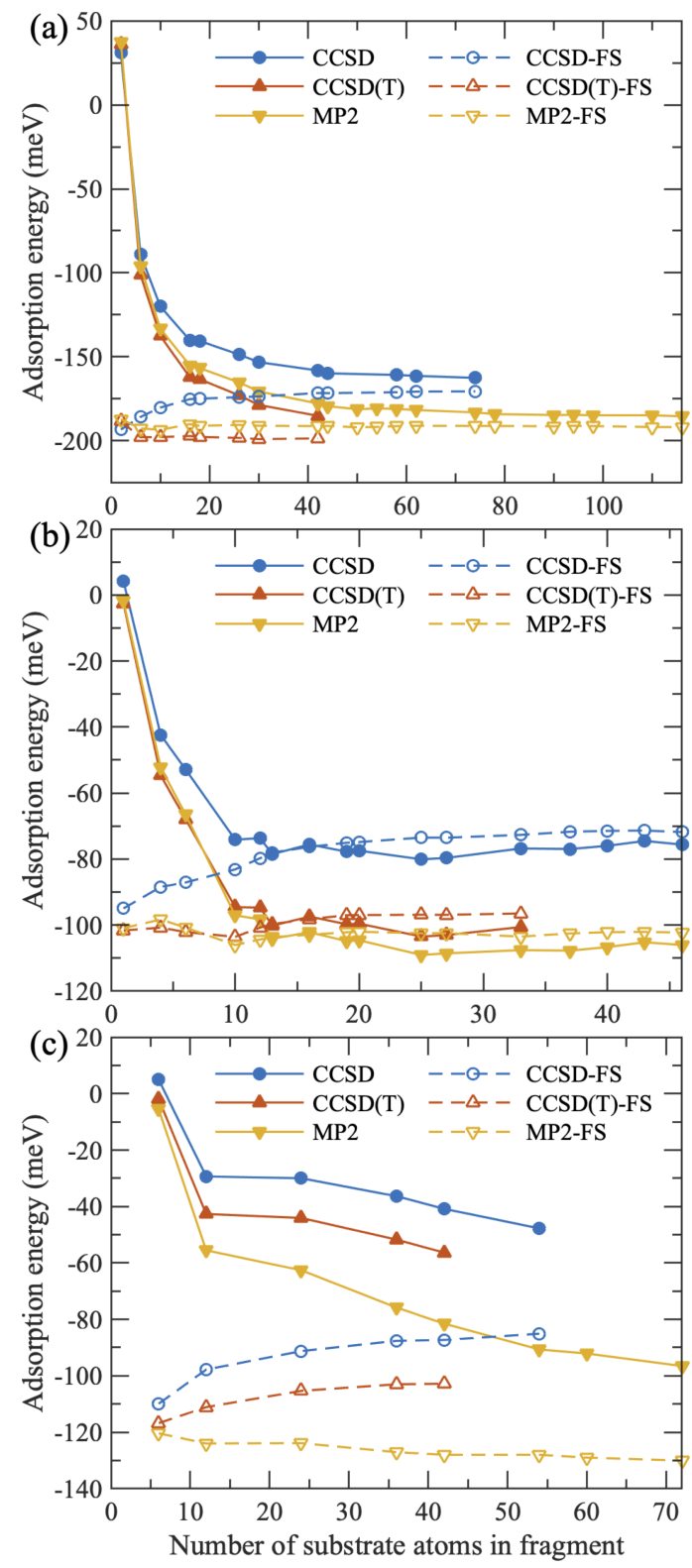

FIG. 3. The post-HF adsorption energy as a function of fragment size, for water on (a) $6 \times 6 \mathrm{LiH}$, (b) $9 \times 9 \mathrm{hBN}$, and (c) $8 \times 8$ graphene, with TZVP on the substrate fragment atoms and DZVP on the rest of the substrate. The solid lines with filled symbols correspond to uncorrected values, while the dashed lines with open symbols correspond to finite-size (FS) corrected values, as described in the text.

\begin{tabular}{ccccccc}
\hline \hline & \multicolumn{2}{c}{ LiH } & \multicolumn{2}{c}{$\mathrm{hBN}$} & \multicolumn{2}{c}{ graphene } \\
& present & lit. $^{32}$ & present & lit. & present & lit. $^{36}$ \\
\hline MP2 & 192 & 233 & 102 & $110,^{33} 118^{6}$ & 130 & - \\
CCSD & 171 & 229 & 72 & $84^{6}$ & 85 & - \\
CCSD(T) & 199 & 254 & 97 & $103^{6}$ & 103 & 87 \\
\hline \hline
\end{tabular}

TABLE I. Adsorption energy of a water molecule on LiH, hBN, and graphene, comparing results obtained in the present manuscript and in the literature (lit.). All energies are in $\mathrm{meV}$.
Considering differences in implementation details (e.g. pseudopotentials) and that we have not attempted a complete basis set limit extrapolation, which can increase the adsorption energy by as much as $20 \mathrm{meV},{ }^{32}$ our results compare favorably to recent literature values, $, 32,33,36$ as shown in Table I. We emphasize that previous calculations for all three systems have only been performed on $4 \times 4$ supercells and then extrapolated to correct for finite-size effects. In contrast, our approach allows us to directly simulate much larger supercells up to $9 \times 9$, and monitor the convergence as a function of the number of atoms in the local fragment. Taken together, the combination of regional embedding and focal point corrections (for basis set and finite-size effects) enables the calculation of high-quality ab initio adsorption energies at very low computational cost.

In summary, we have presented regional embedding, which allows an isolated treatment of local chemical changes with high level wave function methods. This is achieved by unitary transformations of the occupied and virtual Hartree-Fock orbitals based on the degree of overlap with the fragment's Hilbert space. The conventional use of high level wave functions in sufficiently large supercells is expensive. In contrast, regional embedding restricts the correlated calculation to the Hilbert space associated with the target fragment; consequently, large supercells can be used and the finite-size error can be efficiently eliminated. The benchmarks presented indicate that regional embedding with finite-size (focal point) corrections allows remarkably quick convergence to the thermodynamic limit, although the rate of convergence depends on the nature of molecule-substrate interaction. Converged results are achieved with correlated calculations on much smaller systems than the supercells used in contemporary periodic quantum chemistry and quantum Monte Carlo methods.

We expect this method to have broad applicability in molecular, biological, and condensed systems. The simplicity and performance should make it an appealing choice whenever modeling localized chemical changes in large systems. This covers many applications scenarios, and we have already started projects in heterogeneous catalysis and in the optical and thermochemical properties crystal defects. Concerning the theory, treating the frozen orbitals at levels beyond focal-point corrections may further increase the power of the method; self-consistent quantum embeddings, canonical transformations, or screened interactions may provide viable avenues. Finally, a core appeal of this method is that the embedded quantum system can be handled with essentially arbitrary many-body solvers, including quantum chemical multireference methods for strongly correlated systems. This may open a pathway to reliable treatments of complex electronic structure phenomena also in condensed phases and at interfaces. For example, these methods can be used to address long-standing problems in catalyst doping, catalysis at surface defects (e.g. kinks and edges), or the surface chemistry of transition metals and radicals. 


\section{ASSOCIATED CONTENT}

\section{Supporting Information}

The Supporting Information is available free of charge at [xxx]. Finite-size extrapolation of MP2 adsorption energies and discussion of the choice of eigenvalue cutoff in the regional embedding projection operators.

\section{ACKNOWLEDGMENTS}

T.C.B. thanks Sandeep Sharma for helpful discussions. This work was supported in part by the NSF CAREER program under award No. CHE-1945276 (G.K.) and by the NSF Cyberinfrastructure for Sustained Scientific Innovation program via award No. OAC-1931258 (T.C.B.). The Flatiron Institute is a division of the Simons Foundation.

\section{REFERENCES}

${ }^{1}$ Marsman, M.; Grüneis, A.; Paier, J.; Kresse, G. Second-Order Møller-Plesset Perturbation Theory Applied to Extended Systems. I. Within the Projector-Augmented-Wave Formalism Using a Plane Wave Basis Set. J Chem Phys 2009, 130, 184103.

${ }^{2}$ Müller, C.; Paulus, B. Wavefunction-based electron correlation methods for solids. Phys. Chem. Chem. Phys. 2012, 14, 7605-7614.

${ }^{3}$ Booth, G. H.; Grüneis, A.; Kresse, G.; Alavi, A. Towards an Exact Description of Electronic Wavefunctions in Real Solids. Nature 2013, 493, 365-370.

${ }^{4}$ Yang, J.; Hu, W.; Usvyat, D.; Matthews, D.; Schütz, M.; Chan, G. K.-L. Ab Initio Determination of the Crystalline Benzene Lattice Energy to SubKilojoule/Mole Accuracy. Science 2014, 345, 640-643.

${ }^{5}$ McClain, J.; Sun, Q.; Chan, G. K.-L.; Berkelbach, T. C. Gaussian-Based Coupled-Cluster Theory for the Ground-State and Band Structure of Solids. J. Chem. Theory Comput. 2017, 13, 1209-1218.

${ }^{6}$ Gruber, T.; Liao, K.; Tsatsoulis, T.; Hummel, F.; Grüneis, A. Applying the Coupled-Cluster Ansatz to Solids and Surfaces in the Thermodynamic Limit. Phys. Rev. X 2018, 8, 021043.

${ }^{7}$ Zhang, I. Y.; Grüneis, A. Coupled cluster theory in materials science. Front. Mater. 2019, 6 .

${ }^{8}$ Dovesi, R. et al. The CRYSTAL code, 1976-2020 and beyond, a long story. J. Chem. Phys. 2020, 152, 204111.

${ }^{9}$ Maurer, R. J.; Ruiz, V. G.; Camarillo-Cisneros, J.; Liu, W.; Ferri, N.; Reuter, K.; Tkatchenko, A. Adsorption structures and energetics of molecules on metal surfaces: Bridging experiment and theory. Prog. Surf. Sci. 2016, $91,72-100$.

${ }^{10}$ Govind, N.; Wang, Y. A.; Carter, E. A. Electronic-Structure Calculations by First-Principles Density-Based Embedding of Explicitly Correlated Systems. The Journal of Chemical Physics 1999, 110, 7677-7688.

${ }^{11}$ Chulhai, D. V.; Goodpaster, J. D. Projection-Based Correlated Wave Function in Density Functional Theory Embedding for Periodic Systems. $J$. Chem. Theory Comput. 2018, 14, 1928-1942.

${ }^{12}$ Virgus, Y.; Purwanto, W.; Krakauer, H.; Zhang, S. Stability, energetics, and magnetic states of cobalt adatoms on graphene. Phys. Rev. Lett. 2014, 113, $1-6$.

${ }^{13}$ Eskridge, B.; Krakauer, H.; Zhang, S. Local Embedding and Effective Downfolding in the Auxiliary-Field Quantum Monte Carlo Method. $J$. Chem. Theory Comput. 2019, 15, 3949-3959.

${ }^{14}$ Knizia, G.; Chan, G. K.-L. Density Matrix Embedding: A Strong-Coupling Quantum Embedding Theory. J. Chem. Theory Comput. 2013, 9, 14281432.
${ }^{15}$ Wouters, S.; Jiménez-Hoyos, C. A.; Sun, Q.; Chan, G. K. A Practical Guide to Density Matrix Embedding Theory in Quantum Chemistry. J. Chem. Theory Comput. 2016, 12, 2706-2719.

${ }^{16}$ Cui, Z.-H.; Zhu, T.; Chan, G. K.-L. Efficient Implementation of Ab Initio Quantum Embedding in Periodic Systems: Density Matrix Embedding Theory. J. Chem. Theory Comput. 2020, 16, 119-129.

${ }^{17}$ Sayfutyarova, E. R.; Sun, Q.; Chan, G. K.-L.; Knizia, G. Automated Construction of Molecular Active Spaces from Atomic Valence Orbitals. $J$. Chem. Theory Comput. 2017, 13, 4063-4078.

${ }^{18}$ Zheng, B.-X.; Chan, G. K.-L. Ground-state phase diagram of the square lattice Hubbard model from density matrix embedding theory. Phys. Rev. B 2016, 93, 035126.

${ }^{19}$ Foster, J. M.; Boys, S. F. Canonical Configurational Interaction Procedure. Rev. Mod. Phys. 1960, 32, 300-302.

${ }^{20}$ Edmiston, C.; Ruedenberg, K. Localized Atomic and Molecular Orbitals. Rev. Mod. Phys. 1963, 35, 457-464.

${ }^{21}$ Pipek, J.; Mezey, P. G. A fast intrinsic localization procedure applicable for ab initio and semiempirical linear combination of atomic orbital wave functions. J. Chem. Phys. 1989, 90, 4916-4926.

${ }^{22}$ Høyvik, I.-M.; Jansik, B.; Jørgensen, P. Orbital localization using fourth central moment minimization. J. Chem. Phys. 2012, 137, 224114.

${ }^{23}$ Høyvik, I.-M.; Kristensen, K.; Kjærgaard, T.; Jørgensen, P. A perspective on the localizability of Hartree-Fock orbitals. Theor. Chem. Acc. 2014, 133, 1417.

${ }^{24}$ Pulay, P. Localizability of dynamic electron correlation. Chem. Phys. Lett 1983, 100, $151-154$

${ }^{25}$ Saebo, S.; Pulay, P. Local Treatment of Electron Correlation. Annu. Rev. Phys. Chem. 1993, 44, 213-236.

${ }^{26} \mathrm{Hampel}, \mathrm{C}$; Werner, H. Local treatment of electron correlation in coupled cluster theory. J. Chem. Phys. 1996, 104, 6286-6297.

${ }^{27}$ Schütz, M.; Werner, H.-J. Low-order scaling local electron correlation methods. IV. Linear scaling local coupled-cluster (LCCSD). J. Chem. Phys. 2001, 114, 661-681.

${ }^{28}$ Claudino, D.; Mayhall, N. J. Automatic Partition of Orbital Spaces Based on Singular Value Decomposition in the Context of Embedding Theories. J. Chem. Theory Comput. 2019, 15, 1053-1064.

${ }^{29} \mathrm{He}, \mathrm{N}$.; Evangelista, F. A. A zeroth-order active-space frozen-orbital embedding scheme for multireference calculations. J. Chem. Phys. 2020, 152, 094107.

${ }^{30}$ Karton, A.; Rabinovich, E.; Martin, J. M.; Ruscic, B. W4 theory for computational thermochemistry: In pursuit of confident sub-kJ/mol predictions. J. Chem. Phys. 2006, 125, 144108.

${ }^{31}$ Booth, G. H.; Tsatsoulis, T.; Chan, G. K.-L.; Grüneis, A. From plane waves to local Gaussians for the simulation of correlated periodic systems. $J$. Chem. Phys. 2016, 145, 084111.

${ }^{32}$ Tsatsoulis, T.; Hummel, F.; Usvyat, D.; Schütz, M.; Booth, G. H.; Binnie, S. S.; Gillan, M. J.; Alfè, D.; Michaelides, A.; Grüneis, A. A comparison between quantum chemistry and quantum Monte Carlo techniques for the adsorption of water on the (001) LiH surface. J. Chem. Phys. 2017, 146, 204108.

${ }^{33}$ Al-Hamdani, Y. S.; Rossi, M.; Alfè, D.; Tsatsoulis, T.; Ramberger, B.; Brandenburg, J. G.; Zen, A.; Kresse, G.; Grüneis, A.; Tkatchenko, A.; Michaelides, A. Properties of the water to boron nitride interaction: From zero to two dimensions with benchmark accuracy. J. Chem. Phys. 2017, $147,044710$.

${ }^{34}$ Voloshina, E.; Usvyat, D.; Schütz, M.; Dedkov, Y.; Paulus, B. On the physisorption of water on graphene: A CCSD(T) study. Phys. Chem. Chem. Phys. 2011, 13, 12041-12047.

${ }^{35}$ Jenness, G. R.; Karalti, O.; Jordan, K. D. Benchmark calculations of water-acene interaction energies: Extrapolation to the water-graphene limit and assessment of dispersion-corrected DFT methods. Phys. Chem. Chem. Phys. 2010, 12, 6375-6381.

${ }^{36}$ Brandenburg, J. G.; Zen, A.; Fitzner, M.; Ramberger, B.; Kresse, G.; Tsatsoulis, T.; Grüneis, A.; Michaelides, A.; Alfè, D. Physisorption of Water on Graphene: Subchemical Accuracy from Many-Body Electronic Structure Methods. J. Phys. Chem. Lett. 2019, 10, 358-368.

${ }^{37}$ Jordan, K. D.; Heßelmann, A. Comment on "Physisorption of Water on Graphene: Subchemical Accuracy from Many-Body Electronic Structure Methods". J. Phys. Chem. C 2019, 123, 10163-10165. 
${ }^{38}$ Boys, S.; Bernardi, F. The calculation of small molecular interactions by the differences of separate total energies. Some procedures with reduced errors. Mol. Phys. 1970, 19, 553-566.

${ }^{39}$ Sun, Q.; Berkelbach, T. C.; Blunt, N. S.; Booth, G. H.; Guo, S.; Li, Z.; Liu, J.; McClain, J. D.; Sayfutyarova, E. R.; Sharma, S.; Wouters, S.; Chan, G. K.-L. PySCF: the Python-based simulations of chemistry framework. Wires. Comput. Mol. Sci. 2018, 8, e1340.

${ }^{40}$ Sun, Q. et al. Recent developments in the PySCF program package. $J$. Chem. Phys. 2020, 153, 024109.

${ }^{41}$ Sun, Q.; Berkelbach, T. C.; McClain, J. D.; Chan, G. K.-L. Gaussian and plane-wave mixed density fitting for periodic systems. J. Chem. Phys. 2017, $147,164119$.
${ }^{42}$ Goedecker, S.; Teter, M.; Hutter, J. Separable dual-space Gaussian pseudopotentials. Phys. Rev. B 1996, 54, 1703-1710.

${ }^{43}$ Maslen, P. E.; Ochsenfeld, C.; White, C. A.; Lee, M. S.; Head-Gordon, M. Locality and Sparsity of Ab Initio One-Particle Density Matrices and Localized Orbitals. J. Phys. Chem. A 1998, 102, 2215-2222.

${ }^{44}$ Marzari, N.; Mostofi, A. A.; Yates, J. R.; Souza, I.; Vanderbilt, D. Maximally localized Wannier functions: Theory and applications. Rev. Mod. Phys. 2012, 84, 1419-1475.

${ }^{45}$ Souza, I.; Marzari, N.; Vanderbilt, D. Maximally localized Wannier functions for entangled energy bands. Phys. Rev. B 2001, 65, 035109. 


\section{Supporting Information: Regional Embedding Enables High-Level Quantum Chemistry for Surface Science}

Bryan T. G. Lau, ${ }^{1}$ Gerald Knizia, ${ }^{2}$ a) and Timothy C. Berkelbach ${ }^{1,3, b)}$

${ }^{1)}$ Center for Computational Quantum Physics, Flatiron Institute, New York, New York 10010 USA

${ }^{2)}$ Department of Chemistry, Pennsylvania State University, University Park, Pennsylvania 16802 USA

${ }^{3)}$ Department of Chemistry, Columbia University, New York, New York 10027 USA

\section{EXTRAPOLATION OF MP2 ADSORPTION ENERGY CURVES}

Figure 1 plots the supercell MP2 adsorption energies for the systems studied in the manuscript, using the DZVP basis on the substrate. We compare fits of the energies to a linear $1 / N$ and quadratic $1 / N^{2}$ form to extrapolate the results to the thermodynamic, low-density limit. We see that $1 / N$ extrapolation fits our data best, although different extrapolations produce very similar adsorption energies, differing by only 5-8 $\mathrm{meV}$.
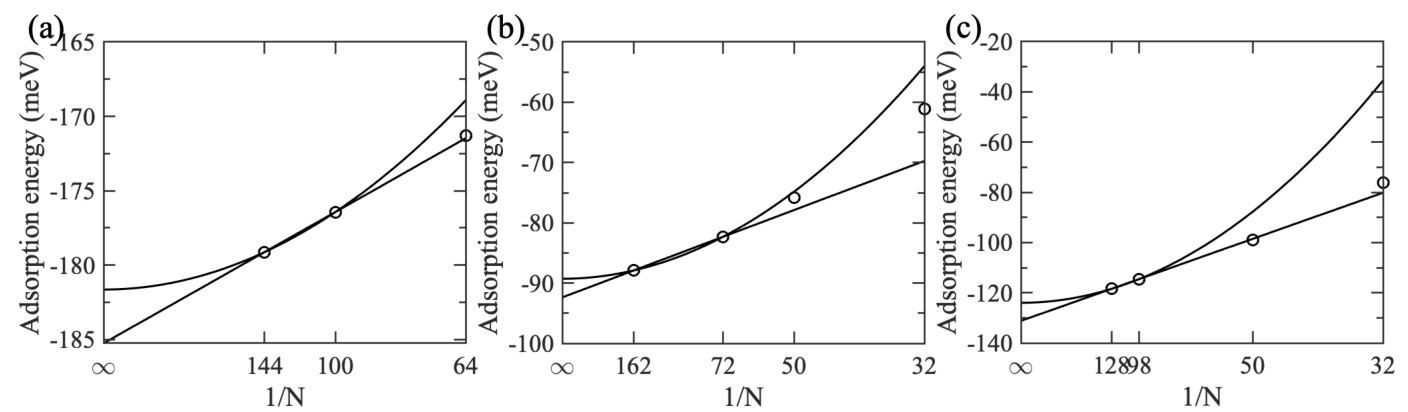

FIG. 1. The supercell MP2 adsorption energies for (a) LiH, (b) hBN, and (c) graphene. The energies are fit to a linear and quadratic form, where $N$ is the number of substrate atoms in the supercell. 